\title{
Caracterización fenotípica y molecular, e influencia de medios de cultivo, en el crecimiento y emisión de luz de bacterias del litoral de La Habana, Cuba
}

\author{
María Victoria Iglesias Rodríguez ${ }^{*}$, Rodolfo Umaña-Castro², Lirialis Garcia Mesa ${ }^{3}$, \\ Eudalys Ortiz Guilarte ${ }^{1}$, Roberto Núñez Moreira ${ }^{1}$, Carlos Álvarez Valcárcel ${ }^{4}$ \\ \& Gladys Margarita Lugioyo Gallardo ${ }^{*}$ \\ 1. Instituto de Ciencias del Mar, Agencia de Medio Ambiente, Ministerio de Ciencia, Tecnología y Medio Ambiente. \\ Calle Loma esq.39, Vedado, La Habana CP 10600, Cuba; victoria@icimar.cu, laly@icimar.cu, robertico@icimar.cu, \\ margarita@icimar.cu \\ 2. Laboratorio de Análisis Genómico, Escuela de Ciencias Biológicas, Campus Omar Dengo, Universidad Nacional, \\ Heredia, Costa Rica; rodolfo.umana.castro@una.ac.cr \\ 3. Centro de Investigaciones Pesqueras, Ministerio de la Industria Alimentaria, Calle 246 e/ 5 ta. Ave. y Mar, Edificio \\ 503, Playa, La Habana, Cuba; lirialis@cip.alinet.cu \\ 4. Centro de Estudios de Proteínas, Facultad de Biología, Universidad de La Habana, Cuba; calvarez@fbio.uh.cu \\ * Correspondencia
}

Recibido 20-III-2020. Corregido 02-VII-2020. Aceptado 04-IX-2020.

\begin{abstract}
Phenotypic and molecular characterization, and influence of culture media, on the growth and light emission of bacteria from the coast of Havana, Cuba. Introduction: The light-emitting enzyme complex of luminescent bacteria is a powerful biochemical tool, with a wide variety of applications including environmental quality monitoring. Objectives: To identify taxonomically two luminescent bacteria from Cuban shelf waters, as well as select the culture media that favor their growth and luminescence. Methods: The taxonomic location of the luminescent bacteria was carried out using traditional and molecular methods. Four culture media (LM, Boss, Chalk, ZoBell) were evaluated as a function of specific growth rate $(\mu)$ and luminescence, using a Genesys 10UV spectrophotometer and a Shimadzu RF-5301pc spectrofluorometer, respectively. Results: Biochemical and physiological characterization of CBM-976 and CBM-992 isolates showed similarities with Vibrio harveyi species. Phylogenetic positioning analysis confirmed a high correspondence with $V$. harveyi strains isolated from aquatic environments, using partial sequences of $16 \mathrm{~S}$ rRNA, gyrB and pyrH genes. LM and ZoBell culture media were selected for having a high specific growth rate of CBM-976 and CBM-992 strains, as well as for showing high luminescence values. The results will allow deepening the physiological characterization and are the starting point for the development of contaminant detection methods. Conclusions: The rational combination of physiological and biochemical characteristics, as well as the molecular approach, contributed to determine the taxonomic position of CBM-976 and CBM-992 strains isolated from Cuban marine waters as Vibrio harveyi. Furthermore, LM and ZoBell culture media were selected as the most suitable for growth and luminescence emission for both strains.
\end{abstract}

Key words: marine bacteria; bioluminescence; Vibrio harveyi; culture media; DNA sequencing.

Iglesias Rodríguez, M.V., Umaña Castro, R., Garcia Mesa, L., Ortiz Guilarte, E., Núñez Moreira, R., Álvarez Valcárcel, C., \& Lugioyo Gallardo, G.M. (2020). Caracterización fenotípica y molecular, e influencia de medios de cultivo, en el crecimiento y emisión de luz de bacterias del litoral de La Habana, Cuba. Revista de Biología Tropical, 68(4), 1298-1310. 
La bioluminiscencia es un proceso bioquímico por el que los seres vivos emiten luz. Los organismos luminiscentes se encuentran ampliamente distribuidos a lo largo del planeta y las bacterias luminiscentes marinas se consideran las más abundantes (Medvedeva, Tyulkova, Kuznetsov, \& Rodicheva, 2009). Los factores que afectan la emisión de luz en bacterias resultan más complejos para las de vida libre en el medio marino, ya que los autoinductores responsables de estimular la emisión luminosa rara vez alcanzan la densidad crítica para disparar este fenómeno. En contraste, las que habitan en los órganos luminosos de especies de peces y calamares, tales como el pez Monocentris japonica y el calamar Euprymna scolopes, superan la concentración crítica del inductor con mayor facilidad (Engebrecht \& Silverman, 1987). Asimismo, se ha comprobado que algunas de las bacterias luminiscentes dejan de emitir luz cuando se modifica su entorno, lo que sugiere que la luminiscencia no solo está controlada por la densidad celular, y otros factores intrínsecos, sino también por factores ambientales tales como el $\mathrm{pH}$, la temperatura y la salinidad (Kuts \& Ismailov, 2009). Otros factores exógenos al medio marino, tales como los xenobióticos, pueden afectar sensiblemente la emisión luminosa de lo que deriva su importancia práctica (Beh, Lim, Asmat, Lee, \& Salmijah, 2010).

Dada la sensibilidad de la emisión luminiscente a las condiciones ambientales, las bacterias luminiscentes han sido empleadas en sistemas de monitoreo de la calidad ambiental y en otras aplicaciones (Podgórska, Chêæ, Ulanowska, \& Wêgrzyn, 2005; Podgórska \& Węgrzyn, 2006). El uso de las bacterias luminiscentes como indicadores de toxicidad se fundamenta en que algunos sistemas bioquímicos y fisiológicos se encuentran altamente conservados durante la evolución y determinados xenobióticos actúan de manera análoga en diversos organismos (Girotti, Bolelli, Ferri, Carpené, \& Isani, 2015). Por otro lado, la sensibilidad, reproducibilidad y rapidez, de los ensayos basados en la luminiscencia bacteriana se han recomendado para evaluar la toxicidad de algunos ecosistemas (Parvez, Venkataraman \& Mukherji, 2006).

Los géneros más representativos de bacterias luminiscentes son Vibrio, Photobacterium y Shewanella; donde las especies más comunes son: $V$. harveyi, $V$. fischeri (actualmente propuesta como Alivivibrio fischeri), P. phosphoreum, P. leiognathi y S. hanedai (Martín, Serrano, Santos, Marquina, \& Vázquez, 2010). Estos géneros se encuentran ampliamente distribuidos tanto en zonas costeras como en mar abierto (Sáenz \& Nevárez, 2010). Lugioyo, Bellota y Moreta (1994) reportaron el primer aislamiento de bacterias luminiscentes en Cuba de aguas de la plataforma noroccidental y aguas oceánicas al sur de la Isla. Posteriormente fue identificada una cepa procedente de aguas costeras como Vibrio harveyi CBM-784 (Delgado et al., 2017).

Como parte de la caracterización fisiológica de las bacterias luminiscentes es imprescindible analizar la composición de los diferentes medios de cultivo por su influencia en el crecimiento y particularmente en la emisión de luz. En el caso del medio LM recomendado por Baumann y Baumann (1981) se refiere que el carbonato de calcio es un inductor de la luminiscencia. La disponibilidad en las condiciones de cultivo de determinados componentes como el carbonato de calcio, el glicerol y las sales de magnesio influyen en la emisión de la luminiscencia de este grupo bacteriano (Baumann \& Baumann, 1981; Tabei, Era, Ogawa, \& Morita, 2011).

Teniendo en cuenta las ventajas que ofrece el trabajo con bacterias luminiscentes para la evaluación de la calidad ambiental, este estudio tuvo como objetivos caracterizar taxonómicamente dos cepas de bacterias luminiscentes aisladas de aguas de la plataforma cubana. Además, determinar la influencia de diferentes medios de cultivo en su crecimiento y luminiscencia.

\section{MATERIALES Y MÉTODOS}

Las cepas CBM-976 y CBM-992, depositadas en la Colección de Bacterias Marinas 
(CBM) del Instituto de Ciencias del Mar (ICIMAR), fueron aisladas de la plataforma noroes-

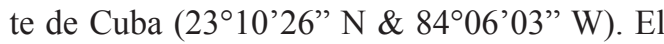
medio empleado para el mantenimiento de estos cultivos fue el LM recomendado para el aislamiento de bacterias luminiscentes (Baumann \& Baumann, 1981).

Identificación taxonómica de los aislados: Para la identificación de las cepas CBM-976 y CBM-992 se ensayaron diferentes pruebas morfológicas, bioquímicas y fisiológicas recomendadas por el Bergey's Manual (De Vos et al., 2009). Se emplearon como referentes para la comparación con los ensayos fisiológicos y bioquímicos la cepa Vibrio harveyi CBM-784 (Delgado et al., 2017) depositada en la CBM y otras ocho bacterias luminiscentes procedentes de la colección de cultivos tipo de Estados Unidos (American Type Culture Collection Catalog) sugeridas por Bagordo et al. (2012). La forma y agrupamiento de las células se determinaron mediante los procedimientos estándar de tinción de Gram. Las pruebas realizadas incluyeron: movilidad; actividad catalasa; oxidasa; emisión de luminiscencia; crecimiento en medio TCBS. Además, en el medio LM se evaluó el crecimiento a diferentes temperaturas $\left(4,10,15,25,30,35\right.$ y $\left.40{ }^{\circ} \mathrm{C}\right)$ y la tolerancia al $\mathrm{NaCl}(0,1,3,6,8$ y $10 \%$ ) (Pedersen et al., 1998).

Se determinó la presencia de las actividades correspondientes a reducción de nitratos, metabolismo óxido-fermentativo de la glucosa $(\mathrm{O} / \mathrm{F})$, la hidrólisis de orto-nitrofenil- $\beta$ D-galactopiranósido (ONPG) y la arginina dihidrolasa (Ramasamy et al., 2014). Se evaluó la producción de $\mathrm{H}_{2} \mathrm{~S}$ a partir de tiosulfato, la reducción de indol, la hidrólisis de esculina y gelatina, la hidrólisis de urea y el uso de citrato, mediante tiras reactivas de API 20 NE (BioMeriux, SA, Francia). Siguiendo los métodos propuestos por Harrigan y McCance (1968) se evaluó la producción de amilasa y lipasa, la reacción de Voges-Proskauer (VP), las actividades lisina (LDC) y ornitina descarboxilasa (ODC). Todas las determinaciones se realizaron con tres repeticiones.
La comparación entre las características fisiológicas de los aislados CBM-976 y CBM992 y las cepas empleadas como referencia, se realizó mediante un análisis de similitud con el programa estadístico Primer 6.1.6. Los resultados de las pruebas fisiológicas realizadas se codificaron como 1 (presencia/positivo), 0 (ausencia/negativo) y 9 (datos no comparables). Las similitudes se determinaron mediante el coeficiente de similitud de BraysCurtis de apareamiento simple y la agrupación empleando métodos aglomerativos jerárquicos. Posteriormente, se realizó un dendrograma con el método de agrupamiento por promedio aritmético de grupos pares no ponderados (UPGMA) (Bagordo et al., 2012).

Se realizó una extracción del ADN genómico de las cepas CBM-976 y CBM-992 para el posicionamiento taxonómico mediante abordajes moleculares usando el método fenol/ cloroformo según Thompson et al. (2005). La reacción de PCR tiempo final y la secuenciación en dos direcciones de los fragmentos parciales de la subunidad pequeña del ARN ribosomal (16S ARNr), la subunidad beta de la girasa $(g y r B)$ y la uridilato quinasa $(p y r H)$ se desarrollaron según Delgado et al. (2017). Las secuencias obtenidas fueron editadas manualmente con el programa Geneious R9 (Biomatters Ltd, Nueva Zelanda) y se identificaron mediante la herramienta básica de búsqueda de similitud local (BLASTn, por sus siglas en inglés) (Altschul, Gish, Miller, Myers, \& Lipman, 1990) de la base de datos NCBI (www. ncbi.nlm.nih.gov/blast) mediante parámetros por defecto. Las secuencias resultantes fueron depositadas en el GenBank (www.ncbi.nlm. nih.gov/genbank).

El análisis de posicionamiento taxonómico entre las secuencias parciales CBM-976, CBM992 y las obtenidas en la base de datos Genbank (Apéndice digital 1) se realizó mediante una topología filogenética multilocus de las regiones $16 \mathrm{~S}$ ARNr, gyrB y pyrH. Primeramente, se elaboraron matrices de secuencias por cada gen y se alinearon con el programa en línea MAFFT 7.0 (http://mafft.cbrc.jp/alignment/server), con el método de refinamiento 
iterativo (G-INS-i) y el parámetro $20 \mathrm{PAM} / \mathrm{k}$ $=2$ (Katoh, Rozewicki, \& Yamada, 2017). Por otro lado, se utilizó el programa Gblocks (Castresana, 2000) para seleccionar bloques conservados mediante parámetros menos estrictos de selección de bloques. Para determinar el mejor modelo de sustitución nucleotídica, las matrices individuales se analizaron con jModelTest 2.1.10 (Darriba, Taboada, Doallo, \& Posada, 2012) con la estrategia de selección mediante el criterio de información de Akaike (AIC) y un $95 \%$ de intervalo de confianza. El árbol de posicionamiento taxonómico se elaboró mediante el algoritmo de máxima verosimilitud (ML) con el programa raxmlGUI versión 1.3 (Silvestro \& Michalak, 2012) con el modelo reversible en tiempo general (GTR-GAMMA+I) y un soporte de los nodos derivado del método Bootstrap (2000 réplicas). Los árboles fueron visualizados y editados con el programa FigTree 1.4 (Rambaut, 2009). Posteriormente, se garantizó que no existieran topologías en conflicto entre los árboles obtenidos individualmente y se procedió a concatenar las matrices alineadas de cada uno de los genes mediante el programa SequenceMatrix v1.7.8 (Vaidya, Lohman, \& Meier, 2011) para obtener una topología multigénica consenso mediante el método de ML.

Influencia de la composición del medio de cultivo sobre el crecimiento y la luminiscencia: Se cultivaron las cepas en cuatro medios recomendados para bacterias luminiscentes marinas: (1) Boss: $30 \mathrm{~g} \mathrm{NaCl}, 1$ $\mathrm{g}$ glicerol, $10 \mathrm{~g}$ peptona bacteriológica, $3 \mathrm{~g}$ extracto de carne (Klein, Walczak, Krasnowska, Blaszczak, \& Lipińska, 1995); (2) Chalk: $30 \mathrm{~g} \mathrm{NaCl}, 5 \mathrm{~g}$ peptona, $3 \mathrm{~g}$ extracto de levadura, $5 \mathrm{~g} \mathrm{CaCO}_{3}, 15 \mathrm{~g}$ glicerol, $1000 \mathrm{ml}$ agua destilada (Shanware, Thakre \& Pande, 2013); (3) LM: $0.3 \%(\mathrm{v} / \mathrm{v})$ de glicerol, 5 g extracto de levadura, $5 \mathrm{~g}$ triptona, $1 \mathrm{~g} \mathrm{CaCO}_{3}, 75 \%$ agua de mar y $25 \%$ agua destilada (Baumann \& Baumann, 1981) y (4) ZoBell: 5 g peptona bacteriológica, $1 \mathrm{~g}$ extracto de levadura, $0.1 \mathrm{~g}$ $\mathrm{FePO}_{4} \cdot \mathrm{H}_{2} \mathrm{O}, 75 \%$ agua de mar y $25 \%$ agua destilada (Oppenheimer \& ZoBell, 1952), para seleccionar la variante de mayor crecimiento y emisión de la luminiscencia.

Los cultivos se incubaron en zaranda orbital (Infors HT Ecotron ${ }^{\circledR}$ ) a $160 \mathrm{r} \cdot \mathrm{min}^{-1}$ y $28 \pm 1$ ${ }^{\circ} \mathrm{C}$; a valores iniciales de $\mathrm{pH}$ y salinidad de 7.0 y $3.5 \%$, respectivamente (Ramahian \& Chandramohan, 1994). El crecimiento bacteriano se determinó mediante la medición de la DO a $620 \mathrm{~nm}$ en un espectrofotómetro Genesys ${ }^{\circledR}$ 10UV. En paralelo, se midió la luminiscencia en un espectrofluorímetro Shimadzu ${ }^{\circledR}$ RF$5301 \mathrm{pc}$ en el cual la fuente de luz de excitación se fijó en $0 \mathrm{~nm}$ para evitar la interferencia de luz de la lámpara. Las mediciones se realizaron cada dos horas por un período de $8 \mathrm{~h}$.

Los valores de máxima emisión luminiscente se calcularon a partir de la integración de los espectros de emisión obtenidos entre $400 \mathrm{y}$ $600 \mathrm{~nm}$, mediante los programas Fluorescence Spectroscopy, Optima 2.20R2 y Origin 8.0, fueron expresados como ual $\mathrm{ml}^{-1}$ (unidades arbitrarias de luz por $\mathrm{ml}$ ). La velocidad específica de crecimiento $(\mu)$ se calculó en la fase exponencial mediante el modelo de crecimiento no restringido para caracterizar los aislados en los medios de cultivo empleados (Gódia \& López, 1998). Para comparar la velocidad específica de crecimiento de los aislados y la luminiscencia, una vez que se verificó el cumplimiento de la distribución normal de los datos según Kolmogorov Smirnov y la homogeneidad de varianza por el método de Bartlett, se realizó el análisis de varianza de clasificación simple y la prueba de comparación de medias de rangos múltiples de Duncan (Lerch, 1977) en la cual las diferencias entre las medias se distinguen por diferentes letras. Los resultados fueron procesados con el paquete estadístico Spss v.21.0. Todas las determinaciones se realizaron por triplicado y los resultados se expresaron en términos de la media \pm desviación estándar (DS).

\section{RESULTADOS}

Identificación taxonómica de los aislados: Los aislados de las cepas CBM-976 y CBM-992 presentaron características 
de cultivo muy similares, con colonias amarillo claro, brillantes, translúcidas, redondas y con bordes regulares. Además, emitieron una luz azul-verdosa que se aprecia a simple vista al colocar estas placas en la oscuridad. Por otra parte, la observación microscópica de los cultivos reveló la presencia de bacilos Gram negativos y móviles.

Los resultados de las pruebas bioquímicas y fisiológicas realizadas a los cultivos aparecen en el apéndice digital (Apéndice digital 2). Las cepas bacterianas evaluadas presentaron, al igual que la cepa de referencia $V$. harveyi ATCC 14126, respiración aeróbica facultativa y produjeron enzimas con actividad gelatinolítica, lisina carboxilasa, lipasa frente a Tween 80, caseinasa y amilasa. Los dos cultivos fueron capaces de reducir nitrato y crecieron entre 15 y $40{ }^{\circ} \mathrm{C}$, pero no a $4{ }^{\circ} \mathrm{C}$. Sin embargo, las cepas no produjeron indol ni ornitina carboxilasa, características que las diferencian de la cepa $V$. harveyi ATCC 14126 empleada como referencia. Además, ambas cepas (CBM-976 y CBM992) presentaron cambios de coloración de verde azulado a amarillo en el medio selectivo y diferencial para Vibrio (TCBS).

El dendrograma correspondiente a la clasificación numérica, a través de la cual se compararon las características fisiológicas de los aislados como caracteres independientes, evidenció el mayor porcentaje de similitud entre los aislados de cepas CBM-976 y CBM-992 (96.9\%). Asimismo, pudo apreciarse un $100 \%$ de similitud entre CBM-992 y Vibrio harveyi

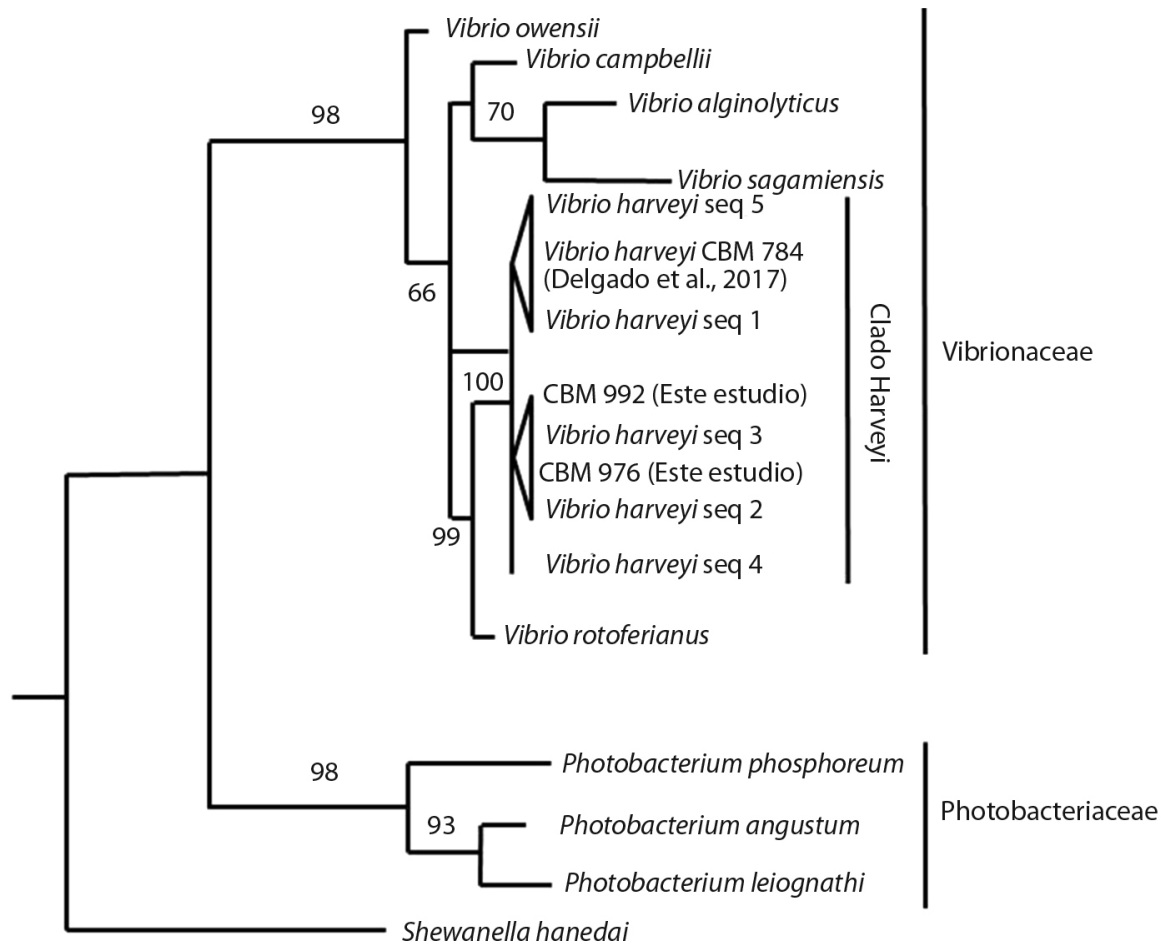

Fig. 1. Árbol de posicionamiento taxonómico de bacterias luminiscentes mediante máxima verosimilitud (ML) y basado en secuencias parciales de la subunidad pequeña del ARN ribosomal (16S ARNr), subunidad beta de la girasa (gyrB) y del gen uridilato quinasa $(\mathrm{pyrH})$ en una topología concatenada. Las cepas de este estudio se definen como CBM-976 y CBM-992. El número en las ramas indica el valor de soporte boostrap (porcentaje derivado de 2000 repeticiones). Shewanella hanedai se estableció como grupo externo.

Fig. 1. Taxonomic positioning tree of luminescent bacteria by maximum likelihood (ML) and based on partial sequences of the ribosomal RNA (16S rRNA) small subunit, beta subunit of gyrB and the uridylate kinase gene (pyrH) in a concatenated topology. The strains in this study are defined as CBM-976 and CBM-992. The number in branches indicates the boostrap support value (percentage derived from 2000 replicates). Shewanella hanedai was set as an external group. 
CBM-784 (Delgado et al., 2017). Por su parte, el conjunto de las cepas CBM-976, CBM-992 y Vibrio harveyi CBM-784 (Delgado et al., 2017) mostraron un $84.4 \%$ de similitud con respecto a la cepa de referencia $V$. harveyi ATCC 14126 (Apéndice digital 3). A través de la identificación basada en las características fisiológicas y bioquímicas se concluyó que las cepas CBM-976 y CBM-992 pertenecen a la especie Vibrio harveyi.

Mediante el análisis de posicionamiento taxonómico multigénico y concatenado, empleando los genes ARNr 16S, gyrB y pyrH, se confirmó la identidad de los aislados CBM976 y CBM-992 como Vibrio harveyi. Se observó que los aislados de este estudio se agrupan como bacterias del género Vibrio con un soporte del nodo de un $98 \%$. Las cepas CBM-976 y CBM-992 se posicionaron en el clado Harveyi (100 \%) y compartieron su posición taxonómica con el aislado de Vibrio rotiferianus con un soporte del nodo del $99 \%$. Por otro lado, la familia Photobacteriaceae, reveló una separación evidente entre los cultivos identificados dentro del grupo Vibrio (Fig. 1). Las secuencias obtenidas en este estudio fueron depositadas en la base de datos del GenBank con los números de acceso correspondientes para cada gen (MT269308 y MT269307, secuencias parciales del gen $16 \mathrm{~S}$ de $1400 \mathrm{pb}$; MT274009 y MT274008, secuencias del gen gyrB de 539 pb; MT274011 y MT274010 secuencias del gen de $p y r H$ de $585 \mathrm{pb}$ ).

Influencia de diferentes medios de cultivo sobre el crecimiento y la luminiscencia de las cepas CBM-976 y CBM-992. La selección de los medios que favorecen el crecimiento y la luminiscencia de los aislados se realizó a partir del análisis del crecimiento microbiano y el comportamiento de la emisión de luminiscencia en el tiempo para cada uno de los medios de cultivos evaluados (Boss, Chalk, LM y ZoBell) (Fig. 2). En general, para las dos cepas y los cuatro medios de cultivo, el patrón de crecimiento fue similar, no se observó la fase de latencia y la fase exponencial se prolongó aproximadamente durante $6 \mathrm{~h}$ (Fig. 2A y 2B).

$\mathrm{Al}$ analizar los valores de velocidad específica de crecimiento $(\mu)$ para ambas cepas en cada uno de los medios de cultivo ensayados, se demostró que para el cultivo CBM-976
CBM-976
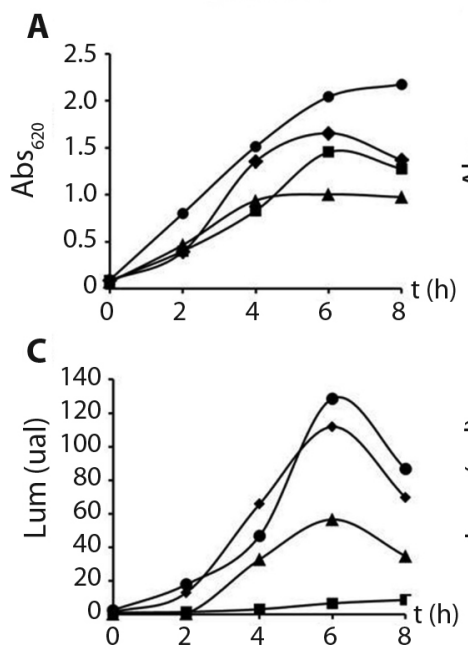

CBM-992
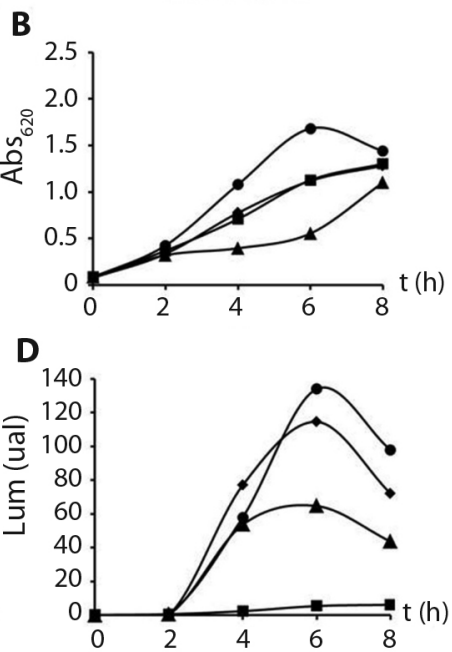

Fig. 2. Influencia del medio de cultivo en el crecimiento (A: CBM-976 y B: CBM-992) y la luminiscencia (C: CBM-976 y D: CBM-992) de los aislados cultivados a $28 \pm 2{ }^{\circ} \mathrm{C}$, $\mathrm{pH} 7$ y 160 r·min ${ }^{-1}$ (medios de cultivo: $\boldsymbol{\bullet}$ Boss, $\boldsymbol{\Delta}$ Chalk, LM y - ZoBell).

Fig. 2. Influence of culture medium on growth (A: CBM-976 and B: CBM-992) and luminescence (C: CBM-976 and D:

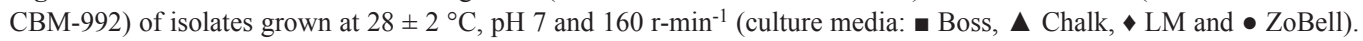


no existen diferencias significativas entre ellos $(\mathrm{P}>0.05)$. Mientras que para la cepa CBM-992, la $\mu$ en el medio Chalk fue menor y significativamente diferente con relación a medios de cultivo Boss, LM y ZoBell (Apéndice digital 4).

Las emisiones máximas de luz para los medios LM, Chalk y ZoBell se produjeron a las $6 \mathrm{~h}$ de cultivo, y resultaron significativamente superiores al final de la fase exponencial e inicio de la fase estacionaria en los medios LM y ZoBell, tanto para CBM-976 como para CBM992 (Fig. 2C y 2D).

Los espectros de emisión obtenidos para todas las condiciones evaluadas presentaron máximos de luminiscencia a $476 \pm 5$ y a $483 \pm$ 3 nm para CBM-976 y CBM-992, respectivamente. En ambos cultivos los mayores valores de luminiscencia se obtuvieron en los medios LM y ZoBell y fueron significativamente diferentes a la emisión de luz encontrada en los medios Boss y Chalk. La luminiscencia máxima en LM y Zobell resultó aproximadamente el doble con respecto a la de Chalk y aproximadamente 25 veces superior a la alcanzada en Boss (Apéndice digital 4).

\section{DISCUSIÓN}

Los resultados confirman que la combinación de métodos para la identificación de bacterias es lo más adecuado. Por un lado, los métodos tradicionales de identificación de bacterias aun siendo menos sensibles, son ampliamente utilizados ya que brindan información sobre la morfología y la actividad de las cepas en cuestión (Fang et al., 2019). Por otro la práctica recomienda complementar los resultados de la caracterización fisiológica y bioquímica con los análisis filogenéticos de secuencias multilocus para la identificación de bacterias luminiscentes (Ast \& Dunlap, 2005; Thompson, et al., 2005; Delgado et al., 2017).

Los abordajes moleculares realizados en este estudio muestran que los aislados se posicionan de manera robusta con el nodo que agrupa a los Vibrio harveyi obtenidos de ambientes $\mathrm{u}$ organismos acuáticos; en tanto discrimina a especies relacionadas tales como $V$. campbellii,
V. owensii, V. sagamiensis, V. alginolyticus, pero no a $V$. rotiferianus, de manera similar a los hallazgos de Ke et. al. (2017) que fueron obtenidos mediante un análisis de genómica comparativa del clado Harveyi, demostrando una alta diversidad genética intra-clado.

Sin embargo, en términos fenotípicos, las cepas CBM-976 y CBM-992 presentaron algunas características diferentes a la cepa $V$. harveyi ATCC 14126; tales como no producir indol ni mostrar actividad ornitina descarboxilasa. Aun cuando las cepas pertenecen a la misma especie, las condiciones ambientales y adaptación en los ecosistemas de procedencia influyen en el metabolismo bacteriano lo que posiblemente contribuya a la aparición de variaciones fenotípicas. Esta variabilidad pudiera atribuirse a la amplia distribución geográfica de las bacterias luminiscentes que condiciona su necesidad adaptativa, así un elevado número de aislados son provenientes de zonas templadas (Bagordo et al., 2012; Dunlap, 2014), en tanto otras han sido obtenidos de zonas tropicales (Martín et al., 2010; Delgado et al., 2017; Rojas-Alfaro, Umaña-Castro, Rojas-Campos, \& Vargas-Montero, 2020).

Las variaciones detectadas en la identificación de especies pertenecientes al grupo Harveyi ( $V$. harveyi, $V$. rotiferianus, $V$. campbellii, $V$. owensii, y V. jasicida) (Cano-Gómez, Høj, Owens, Baillie, \& Andreakis, 2015), mediante herramientas bioquímicas y genéticas, se evidencia en la heterogeneidad fenotípica del grupo (Alsina \& Blanch, 1994). La variabilidad en las características bioquímicas de $V$. harveyi también podría estar relacionada con la transferencia de genes que ocurre, tanto dentro de la misma especie, como entre diferentes géneros bacterianos (Lal \& Ransangan, 2013). Una prueba de ello es la transferencia horizontal de genes lux que tiene lugar de Vibrios luminiscentes $(V$. harveyi) a no luminiscentes $(V$. vulnificus y $V$. chagasii) (Urbanczyk, Ogura, \& Hayashi, 2014). En esta dirección, se ha comprobado que este grupo contiene elementos genéticos móviles (Cano-Gómez, Høj, Owens, \& Andreakis, 2011) y ocurren importantes eventos de recombinación entre las diferentes 
especies (Urbanczyk et al., 2014), lo que contribuye a la adquisición de nuevas características fenotípicas.

Algunos estudios basados en la caracterización fenotípica de bacterias luminiscentes informan a $V$. harveyi, $V$. fischeri y $P$. leiognathi como especies frecuentemente encontradas en aguas costeras templadas y tropicales (Chiu, Chou, Jean, \& Shieh, 2007). En Cuba, han sido descritas especies de Photobacterium phosphoreum, $V$. harveyi y $V$. splendidus en las aguas oceánicas al sur de la Isla, mientras en las aguas de la plataforma noroccidental fueron aisladas las especies P. leiognathi (Lugioyo, 2003) y $V$. harveyi (Delgado et al., 2017).

En los estudios de fisiología bacteriana uno de los aspectos iniciales a tener en cuenta es la selección del medio de cultivo. En particular, para las bacterias luminiscentes se han descrito diversos medios de cultivo con el fin de evaluar el crecimiento y la emisión de luz (Baumann \& Baumann, 1981; Ramahian \& Chandramohan, 1994; Danyluk, Uchman, Konieczny, \& Bilska, 2007).

En los medios de cultivo Chalk, LM y ZoBell, las máximas intensidades de luz de las cepas CBM-976 y CBM-992 se obtuvieron durante el final de la fase exponencial e inicio de la fase estacionaria y son consistentes con lo informado en otras investigaciones (Meighen, 1993; Ramahian \& Chandramohan, 1994; Soto \& Gutierrez, 2009). Coincidentemente, Krieg y Holt (1984) encontraron que la máxima luminiscencia se obtuvo en el inicio de la fase estacionaria del crecimiento y luego disminuyó rápidamente. Otros estudios han demostrado que cuando se alcanza cierto nivel de luminiscencia la síntesis de la enzima finaliza y comienza la inactivación de esta (Soto \& Gutierrez, 2009).

Los máximos de luminiscencia de las cepas CBM-976 y CBM-992 (476 \pm 5 y $483 \pm 3$ $\mathrm{nm}$, respectivamente) se presentaron en las longitudes de onda referidas para bacterias luminiscentes marinas (470-490 nm) (Makiguchi et al., 1979; Dunlap, 2014). Específicamente, en cepas de $V$. harveyi aisladas del Mar Arábigo presentaron los máximos de luminiscencia en el rango entre 466-474 nm (Ramahian \& Chandramohan, 1994).

La emisión de luz en las condiciones experimentales para las dos cepas resultó superior en los medios de cultivo ZoBell y LM. Ambos medios incluyen en su composición un $75 \%$ de agua de mar, que aporta micronutrientes que pudieran estar involucrados como cofactores en el proceso de emisión de luz. En contraste, los medios Boss y Chalk se preparan con agua destilada y $3 \%$ de $\mathrm{NaCl}$, por lo que carecen de otros microelementos. El fenómeno de inducción de la luminiscencia requiere que los medios de cultivo contengan $\mathrm{Na}^{+}, \mathrm{Ca}^{2+}, \mathrm{Cl}^{+}$, $\mathrm{HCO}_{3}^{-}, \mathrm{K}^{+}, \mathrm{Mg}^{2+}$ y S ${ }^{2-}$ (Tabei et al., 2011; Tabei, Era, Ogawa, \& Morita, 2012). En particular, el $\mathrm{Na}^{+}$garantiza en Vibrio fisheri funciones celulares generales tales como la estabilización de actividades de la membrana celular y garantiza la motilidad del flagelo. Asimismo, este ion participa en la cadena respiratoria dependiente de $\mathrm{Na}^{+}$presente en bacterias marinas muy relacionada con el fenómeno de la luminiscencia (Tabei et al., 2011, Tabei et al., 2012). Por otra parte, el medio LM contiene $1 \mathrm{~g} \cdot \mathrm{L}^{-1} \mathrm{de} \mathrm{CaCO}_{3}$, un inductor de la luminiscencia y activador de la respiración dependiente de $\mathrm{Na}^{+}$(Baumann \& Baumann, 1981; Tabei et al., 2012). En especies luminiscentes como Photobacterium phosphoreum, se ha demostrado que el $\mathrm{K}^{+}$contribuye a la expresión de genes involucrados en el fenómeno de bioluminiscencia (Watanabe, Inaba, \& Hastings, 1991). En el medio Chalk, la menor emisión de luz pudiera atribuirse, tanto a la ausencia de micronutrientes como a una alta concentración de glicerol $\left(10 \mathrm{~g} \cdot \mathrm{L}^{-1}\right)$, con respecto al medio LM $\left(3 \mathrm{~g} \cdot \mathrm{L}^{-1}\right)$. Resultados similares se obtuvieron para una cepa de Vibrio rotiferianus aislada de la playa Dui, India, donde en los medios de cultivo Chalk y Boss los niveles de luminiscencia fueron menores que en LM (Shanware et al., 2013).

En esta investigación se corroboró que la combinación de las características fisiológicas y bioquímicas, así como las técnicas basadas en la biología molecular resultaron adecuadas para ubicar la posición taxonómica de las cepas CBM-976 y CBM-992 aisladas 
de aguas marinas cubanas como Vibrio harveyi. Además, se lograron seleccionar los medios LM y ZoBell como los más adecuados para el crecimiento y emisión de la luminiscencia en ambas cepas. A partir de la selección de los medios de cultivos LM y ZoBell se continuarán los estudios de optimización de las condiciones de crecimiento y luminiscencia para las cepas CBM-976 y CBM-992, imprescindibles para el diseño y desarrollo de métodos sencillos y rápidos destinados a la detección de contaminantes en ecosistemas marinos.

Declaración de ética: los autores declaran que todos están de acuerdo con esta publicación y que han hecho aportes que justifican su autoría; que no hay conflicto de interés de ningún tipo; y que han cumplido con todos los requisitos y procedimientos éticos y legales pertinentes. Todas las fuentes de financiamiento se detallan plena y claramente en la sección de agradecimientos. El respectivo documento legal firmado se encuentra en los archivos de la revista.

\section{AGRADECIMIENTOS}

A Carolina Sancho-Blanco por el soporte técnico en los análisis moleculares y de secuenciación Sanger de las cepas bacterianas empleadas en este estudio. Al Centro de Estudios de Proteínas de la Facultad de Biología de la Universidad de La Habana por el apoyo en el desarrollo de la investigación.

\section{RESUMEN}

Introducción: El complejo enzimático emisor de luz de las bacterias luminiscentes es una poderosa herramienta bioquímica, con una amplia variedad de aplicaciones, incluyendo el control de la calidad ambiental. Objetivos: Identificar taxonómicamente dos bacterias luminiscentes de las aguas de la plataforma cubana, así como seleccionar los medios de cultivo que favorezcan su crecimiento y luminiscencia. Métodos: La identificación taxonómica de las bacterias luminiscentes se llevó a cabo utilizando métodos tradicionales y moleculares. Cuatro medios de cultivo (LM, Boss, Chalk, ZoBell) fueron evaluados en función de la tasa de crecimiento específico $(\mu)$ y la luminiscencia utilizando un espectrofotómetro Genesys 10UV y un espectrofluorómetro Shimadzu RF-5301pc, respectivamente. Resultados: La caracterización bioquímica y fisiológica de los aislamientos de CBM-976 y CBM-992 mostró similitudes con las especies de Vibrio harveyi. El análisis del posicionamiento taxonómico confirmó una alta correspondencia con las cepas de $V$. harveyi aisladas de entornos acuáticos, utilizando secuencias parciales de los genes 16S rRNA, gyrB y pyrH. Se seleccionaron los medios de cultivo LM y ZoBell por tener una alta tasa de crecimiento específico de las cepas CBM-976 y CBM-992; así como por mostrar altos valores de luminiscencia. Los resultados permitirán profundizar en la caracterización fisiológica y son el punto de partida para el desarrollo de métodos de detección de contaminantes. Conclusiones: La combinación de las características fisiológicas y bioquímicas, así como las técnicas de biología molecular contribuyeron a determinar la posición taxonómica de las cepas CBM-976 y CBM-992 aisladas de las aguas marinas cubanas como Vibrio harveyi. Además, se seleccionaron los medios de cultivo LM y ZoBell como los más adecuados para el crecimiento y la emisión de luminiscencia de ambas cepas.

Palabras clave: bacteria marina; bioluminiscencia; Vibrio harveyi; medios de cultivo; secuenciación ADN.

\section{REFERENCIAS}

Alsina, M., \& Blanch, A.R. (1994). A set of keys for biochemical identification of environmental Vibrio species. Journal of Applied Bacteriology, 76, 79-85.

Altschul, S.F., Gish, W., Miller, W., Myers, E.W., \& Lipman, D.J. (1990). Basic local alignment search tool. Journal of Molecular Biology, 21, 403-410.

Ast, J.C., \& Dunlap, P.V. (2004). Phylogenetic analysis of the lux operon distinguishes two evolutionarily distinct clades of Photobacterium leiognathi. Archives of Microbiology, 181, 352-361. DOI: 10.1007/ s00203-0040663-7

Ast, J.C., \& Dunlap, P.V. (2005). Phylogenetic resolution and habitat specificity of the Photobacterium phosphoreum species group. Environmental Microbiology, 7, 1641-1654.

Bagordo, F., Serio, F., Lugoli, F., Grassi, T., Idolo, A., Gabutti, G., \& De Donno, A. (2012). Phenotypic characterization of culturable marine luminous bacteria isolated from coastal waters of the southern Adriatic Sea (Otranto, Italy). Ciencias Marinas, 38(4), 599608. DOI: $10.7773 / \mathrm{cm} . v 38 \mathrm{i} 4.2119$

Baumann, P., \& Baumann, L. (1981). The marine Gramnegative eubacteria: genera Photobacterium, Beneckea, Alteromonas, Pseudomonas, \& Alcaligenes. The Prokaryotes. A handbook on habitats, isolation, \& identification of bacteria. Berlin, Germany: Springer-Verlag. 
Beh, W.C., Lim, Y.K., Asmat, A., Lee, Y.H., \& Salmijah, S. (2010). The potential of luminescent Bacteria "Photobacterium leiognathi" as biosensor for the detection of aquatic toxicity. Enviroment and Natural Resources Journal, 8(3), 1-9.

Cano-Gómez, A., Høj, L., Owens, L., \& Andreakis, N. (2011). Multilocus sequence analysis provides basis for fast and reliable identification of Vibrio harveyirelated species and reveals previous misidentification of important marine pathogens. Systematic and Applied Microbiology, 34, 561-565.

Cano-Gómez, A., Høj, L., Owens, L., Baillie, B.K., \& Andreakis, N. (2015). A multiplex PCR-based protocol for identification and quantification of Vibrio harveyi-related species. Aquaculture, 437, 195-200.

Castresana, J. (2000). Selection of conserved blocks from multiple alignments for their use in phylogenetic analysis. Molecular Biology and Evolution, 17(4), 540-552.

Chiu, H.H., Chou, H.H., Jean, W.D., \& Shieh, W.Y. (2007). Isolation and characterization of marine luminous bacteria from shallow coastal waters of Taiwan. Journal of Microbiology, Immunology and Infection, 40, 14-23.

Danyluk, B., Uchman, W., Konieczny, P., \& Bilska, A. (2007). An objective method to assess bioluminescent properties of selected bacterial strains. Acta Scientiarum Polonorum Technologia Alimentaria, 6(4), 5-16.

Darriba, D., Taboada, G.L., Doallo, R., \& Posada, D. (2012). jModelTest 2: more models, new heuristics and parallel computing. Nature Methods, 9(8), 772.

De Vos, P., Garrity, G.M., Jones, D., Krieg, N.R., Ludwig, W., Rainey, F.A., ...Whitman, W.B. (Eds.). (2009). Bergey's manual of Systematic Bacteriology. Second Edition. Volume Three: The Firmicutes. New York, USA: Springer.

Delgado, Y., Umaña, R., Solano, S., Iglesias, M.V., Ortiz, E., Alvarez, C., \& Lugioyo, G.M. (2017). Phenotypic characterization and molecular identification of a luminescent marine bacterium isolated from the NW shelf of Cuba. Biotecnia, 19(3), 3-10.

DeLong, E.F., Franks, D.G., \& Yayanos, A.A. (1997). Evolutionary relationships of cultivated psychrophilic and barophilic deep-sea bacteria. Applied and Environmental Microbiology, 63(5), 2105-2108.

Dorsch, M., Lane, D., \& Stackebrandt, E. (1992). Towards a phylogeny of the genus Vibrio based on 16S rRNA sequences. International Journal of Systematic and Evolutionary Microbiology, 42(1), 58-63.

Dunlap, P. (2014). Biochemistry and genetics of bacterial bioluminescence. In G. Thouand, \& R. Marks (Eds.), Bioluminescence: Fundamentals and Applications in
Biotechnology (Volume 1, pp. 37-64). Berlin, Germany: Springer-Verlag Berlin Heidelberg.

Dunlap, P.V., Ast, J.C., Kimura, S., Fukui, A., Yoshino, T., \& Endo, H. (2007). Phylogenetic analysis of hostsymbiont specificity and codivergence in bioluminescent symbioses. Cladistics, 23(5), 507-532.

Engebrecht, J., \& Silverman, M. (1987). Nucleotide sequence of the regulatory locus controlling expression of bacterial genes for bioluminescence. Nucleic Acids Research, 15(24), 10455-10467.

Fang, Y., Wang, Y., Liu, Z., Dai, H., Cai, H., Li, Z., ... Wei, Q. (2019). Multilocus sequence analysis, a rapid and accurate tool for taxonomic classification, evolutionary relationship determination, and population biology studies of the genus Shewanella. Applied and Environmental Microbiology, 85(11), 3126-3118.

Farto, R., Montes, M., Pérez, M., Nieto, T., Larsen, J., \& Pedersen, K. (1999). Characterization by numerical taxonomy and ribotyping of Vibrio splendidus biovar I and Vibrio scophthalmi strains associated with turbot cultures. Journal of Applied Microbiology, 86(5), 796-804.

Figge, M.J., Robertson, L.A., Ast, J.C., \& Dunlap, P.V. (2011). Historical microbiology: revival and phylogenetic analysis of the luminous bacterial cultures of MW Beijerinck. FEMS Microbiology Ecology, $78(3), 463-472$.

Girotti, S., Bolelli, L., Ferri, E., Carpené, E., \& Isani, G. (2015). Bioindicators in enviromental monitoring: bioluminiscen bacteria, algae and honeybees. Paper presented at the International Conference on Environmental Science and Technology, Rhodes, Greece.

Gódia, F., \& López, J. (1998). Ingeniería Bioquímica (1ra Ed.). España: SINTESIS S.A.

Gomez-Gil, B., Soto-Rodriguez, S., García-Gasca, A., Roque, A., Vazquez-Juarez, R., Thompson, F.L., \& Swings, J. (2004). Molecular identification of Vibrio harveyi-related isolates associated with diseased aquatic organisms. Microbiology, 150(6), 1769-1777.

Harrigan, W.F., \& McCance, M.E. (1968). Métodos de laboratorio en microbiología. España: Editorial Academia.

Jiang, Q., Shi, L., Ke, C., You, W., \& Zhao, J. (2013). Identification and characterization of Vibrio harveyi associated with diseased abalone Haliotis diversicolor. Diseases of Aquatic Organisms, 103(2), 133-139.

Katoh, K., Rozewicki, J., \& Yamada, K.D. (2017). MAFFT online service: multiple sequence alignment, interactive sequence choice and visualization. Briefings in Bioinformatics, 20(4), 1160-1166.

Ke, G., Meng, Q., Finley, T., Wang, T., Chen, W., Ma, W., ... Liu, T.Y. (2017). Lightgbm: A highly efficient 
gradient boosting decision tree. The Advances in neural information processing systems, 3146-3154.

Klein, G., Walczak, R., Krasnowska, E., Blaszczak, A., \& Lipińska, B. (1995). Characterization of heat-shock response of the marine bacterium Vibrio harveyi. Molecular Microbiology, 16(4), 801-811.

Krieg, N.R., \& Holt, J.G. (1984). Bergey’s Manual of Systematic Bacteriology (Vol. 1). Baltimore, London, UK: Williams and Willkins.

Kuts, V., \& Ismailov, A. (2009). Physiological and emission characteristics of the luminescent bacterium Photobacterium phosphoreum from the White Sea. Microbiology, 78(5), 554.

Lal, M.T.B.M., \& Ransangan, J. (2013). Taxonomic classification of Vibrio harveyi using 16S rDNA and atpA gene sequencing method. International Journal of Research in Pure and Applied Microbiology, 3(1), 17-24.

Lerch, G. (1977). La experimentación en las ciencias biológicas y agrícolas. La Habana, Cuba: Editorial Científico Técnica.

López-Caballero, M.E., Torres, M.D.A., Sánchez-Fernández, J.A., \& Moral, A. (2002). Photobacterium phosphoreum isolated as a luminescent colony from spoiled fish, cultured in a model system under controlled atmospheres. European Food Research and Technology, 215, 390-395.

Lugioyo, G.M. (2003). Distribución, relaciones tróficas y diversidad del bacterioplancton de las aguas oceánicas de Cuba (Tesis de Doctorado). Universidad de La Habana, La Habana, Cuba.

Lugioyo, M., Bellota, M., \& Moreta, N. (1994). Primer registro sobre bacterias luminiscentes aisladas de aguas cubanas. Avicennia, 2, 73-82.

Lunder, T., Sørum, H., Holstad, G., Steigerwalt, A.G., Mowinckel, P., \& Brenner, D.J. (2000). Phenotypic and genotypic characterization of Vibrio viscosus sp. nov. and Vibrio wodanis sp. nov. isolated from Atlantic salmon (Salmo salar) with'winter ulcer'. International Journal of Systematic and Evolutionary Microbiology, 50(2), 427-450.

Luo, P., \& Hu, C. (2008). Vibrio alginolyticus gyrB sequence analysis and gyrB-targeted PCR identification in environmental isolates. Diseases of Aquatic Organisms, 82(3), 209-216.

Makemson, J.C., Fulayfil, N.R., Landry, W., Van Ert, L.M., Wimpee, C.F., Widder, E.A., \& Case, J.F. (1997). Shewanella woodyi sp. nov., an exclusively respiratory luminous bacterium isolated from the Alboran Sea. International Journal of Systematic and Evolutionary Microbiology, 47(4), 1034-1039.
Makiguchi, N., Arita, M., \& Asai, Y. (1979). Isolation, identification, and several characteristics of luminous bacteria. The Journal of General and Applied Microbiology, 25, 387-396.

Martín, A., Serrano, S., Santos, A., Marquina, D., \& Vázquez, C. (2010). Bioluminiscencia bacteriana. Reduca (Biología). Serie Microbiología, 3(5), 75-86.

Medvedeva, S.E., Tyulkova, N.A., Kuznetsov, A.M., \& Rodicheva, E.K. (2009). Bioluminescent bioassays based on luminous bacteria. Journal of Siberian Federal University. Biology, 4(2), 418-452. DOI: 574.24: 577.344: 579.843

Meighen, E.A. (1993). Bacterial bioluminescence: organization, regulation and application of the lux genes. Reviews, 1016-1022.

Oppenheimer, C.H., \& ZoBell, C.E. (1952). The growth and viability of sixty three species of marine bacteria as influenced by hydrostatic pressure. Journal of Marine Research, 11, 10-18.

Parvez, S., Venkataraman, C., \& Mukherji, S. (2006). A review on advantages of implementing luminescence inhibition test (Vibrio fischeri) for acute toxicity prediction of chemicals. Environment International, 32(2), 265-268.

Pascual, J., Macián, M.C., Arahal, D.R., Garay, E., \& Pujalte, M.J. (2010). Multilocus sequence analysis of the central clade of the genus Vibrio by using the $16 \mathrm{~S}$ rRNA, rec A, pyrH, rpo D, gyr $\mathrm{B}, r c t \mathrm{~B}$ and tox $\mathrm{R}$ genes. International Journal of Systematic and Evolutionary Microbiology, 60(1), 154-165.

Pedersen, K., Verdonck, L., Austin, B., Austin, D.A., Blanch, A.R., Grimont, P.A., ... Tiainen, T. (1998). Taxonomic evidence that Vibrio carchariae Grimes et al. 1985 is a junior synonym of Vibrio harveyi (Johnson and Shunk 1936) Baumann et al. 1981. International Journal of Systematic and Evolutionary Microbiology, 48(3), 749-758.

Podgórska, B., Chêæ, E., Ulanowska, K., \& Wêgrzyn, G. (2005). Optimisation of the microbiological mutagenicity assay based on genetically modified Vibrio harveyi strains. Journal of Applied Genetic, 46(2), 241-246.

Podgórska, B., \& Węgrzyn, G. (2006). A Modified Vibrio harveyi mutagenicity assay based on bioluminescence induction. Letters in Applied Microbiology, $42,578-582$.

Ramahian, N., \& Chandramohan, D. (1994). Bacterial bioluminescens in marine pollution assessment. Ocean Technology: Perspectives, 967-980.

Ramasamy, D., Mishra, A.K., Lagier, J.C., Padhmanabhan, R., Rossi, M., Sentausa, E., ... Fournier, P.E. (2014). A polyphasic strategy incorporating genomic data for the taxonomic description of novel bacterial 
species. International Journal of Systematic and Evolutionary Microbiology, 64, 384-391. DOI: 10.1099/ ijs.0.057091-0

Rambaut, A. (2009). FigTree v1.4. 2012-2014. Retreived from http://tree.bio.ed.ac.uk/software/figtree

Ravindran, J., Kannapiran, E., Manikandan, B., Francis, K., Arora, S., Karunya, E., ... Jose, J. (2013). UVabsorbing bacteria in coral mucus and their response to simulated temperature elevations. Coral reefs, 32(4), 1043-1050.

Rivera-Posada, J., Pratchett, M., \& Owens, L. (2011). Injection of Acanthaster planci with thiosulfatecitrate-bile-sucrose agar (TCBS). II. Histopathological changes. Diseases of Aquatic Organisms, 97(2), 95-102.

Rojas-Alfaro, R., Umaña-Castro, R., Rojas-Campos, N., \& Vargas-Montero, M. (2020). Primer reporte de bacterias y dinoflagelados marinos luminiscentes del Parque Nacional Isla del Coco, Costa Rica. Revista Biología Tropical, 68(Suppl. 1), S213-S224.

Sáenz, C.I., \& Nevárez, G.V. (2010). La bioluminiscencia de microorganismos marinos y su potencial biotecnológico. Revista Científica de la Universidad Autónoma de Coahuila, 2(3), 1-7.

Satomi, M., Oikawa, H., \& Yano, Y. (2003). Shewanella marinintestina sp. nov., Shewanella schlegeliana sp. nov. and Shewanella sairae sp. nov., novel eicosapentaenoic-acid-producing marine bacteria isolated from sea-animal intestines. International Journal of Systematic and Evolutionary Microbiology, 53(2), 491-499.

Shanware, A., Thakre, N., \& Pande, S. (2013). Isolation and characterization of novel marine luminescent bacteria from Diu beach, India. Journal of Pharmacy Research, 7, 529-533.

Sharma, S., Pradeep, M., Loka, J., Sahoo, A., Dube, P., Sadhu, N., \& Philipose, K.K. (2014). Association of Vibrio harveyi in mortality of mangrove red snapper (Lutjanus argentimaculatus, Forsskål, 1775) cultured in open sea cages. Indian Journal of Fisheries, 61(3), 118-121.

Shen, G., Shi, C., Fan, C., Jia, D., Wang, S., Xie, G., Li, G., Mo, Z., \& Huang, J. (2017). Isolation, identification and pathogenicity of Vibrio harveyi, the causal agent of skin ulcer disease in juvenile hybrid groupers Epinephelus fuscoguttatus $\times$ Epinephelus lanceolatus. Journal of fish diseases, 40(10), 1351-1362.

Silvestro, D., \& Michalak, I. (2012). raxmlGUI: a graphical front-end for RAxML. Organisms Diversity \& Evolution, 12, 335-337.

Soto, W., \& Gutierrez, J. (2009). Salinity and temperature effects on physiological responses of Vibrio fischeri from diverse ecological niches. Microbial Ecology, 57(1), 140-150. DOI: 10.1007/s00248-008-9412-9

Tabei, Y., Era, M., Ogawa, A., \& Morita, H. (2011). Effects of magnesium sulfate on the luminescence of Vibrio fischeri under nutrient-starved conditions. Bioscience, Biotechnology, and Biochemistry, 75(6), 1073-1078.

Tabei, Y., Era, M., Ogawa, A., \& Morita, H. (2012). Interactions between bicarbonate, potassium, and magnesium, and sulfur-dependent induction of luminescence in Vibrio fischeri. Journal of Basic Microbiology, 52(3), 350-359.

Tall, A., Hervio-Heath, D., Teillon, A., Boisset-Helbert, C., Delesmont, R., Bodilis, J., \& Touron-Bodilis, A. (2013). Diversity of Vibrio spp. isolated at ambient environmental temperature in the Eastern English Channel as determined by pyr H sequencing. Journal of Applied Microbiology, 114(6), 1713-1724.

Thompson, F.L., Gevers, D., Thompson, C.C., Dawyndt, P., Naser, S., Hoste, B., ... Swings, J. (2005). Phylogeny and molecular identification of Vibrios on the basis of multilocus sequence analysis. Applied and Environmental Microbiology, 71, 5107-5115.

Thompson, F.L., Gomez-Gil, B., Vasconcelos, A.T.R., \& Sawabe, T. (2007). Multilocus sequence analysis reveals that Vibrio harveyi and V. campbellii are distinct species. Applied and Environmental Microbiology, 73(13), 4279-4285.

Tonon, L.A.C., Silva, B.S.D.O., Moreira, A.P.B., Valle, C., Alves Jr, N., Cavalcanti, G., Garcia, G., Lopes, R.M., Francini-Filho, R.B., \& de Moura, R.L. (2015). Diversity and ecological structure of vibrios in benthic and pelagic habitats along a latitudinal gradient in the Southwest Atlantic Ocean. PeerJ, 3, e741.

Urbanczyk, H., Ast, J.C., Higgins, M.J., Carson, J., \& Dunlap, P.V. (2007). Reclassification of Vibrio fischeri, Vibrio logei, Vibrio salmonicida and Vibrio wodanis as Aliivibrio fischeri gen. nov., comb. nov., Aliivibrio logei comb. nov., Aliivibrio salmonicida comb. nov. and Aliivibrio wodanis comb. nov. International Journal of Systematic and Evolutionary Microbiology, 57(12), 2823-2829.

Urbanczyk, H., Ast, J.C., Kaeding, A.J., Oliver, J.D., \& Dunlap, P.V. (2008). Phylogenetic analysis of the incidence of lux gene horizontal transfer in Vibrionaceae. Journal of Bacteriology, 190(10), 3494-3504.

Urbanczyk, H., Ogura, Y., \& Hayashi, T. (2014). Contrasting inter- and intraspecies recombination patterns in the "Harveyi clade" vibrio collected over large spatial and temporal scales. Genome Biology and Evolution, 7(1), 71-80.

Vaidya, G., Lohman, D.J., \& Meier, R. (2011). SequenceMatrix: concatenation software for the fast assembly 
of multi-gene datasets with character set and codon information. Cladistics, 27(2), 171-180.

Venkateswaran, K., Moser, D.P., Dollhopf, M.E., Lies, D.P., Saffarini, D.A., MacGregor, B.J., ... Sano, H. (1999). Polyphasic taxonomy of the genus Shewanella and description of Shewanella oneidensis sp. nov. International Journal of Systematic and Evolutionary Microbiology, 49(2), 705-724.

Watanabe, H., Inaba, H., \& Hastings, J.W. (1991). Effects of aldehyde and internal ions on bioluminescence expression of Photobacterium phosphoreum. Archives of Microbiology, 156(1), 1-4.
Yoshizawa, S., Karatani, H., Wada, M., \& Kogure, K. (2012). Vibrio azureus emits blue-shifted light via an accessory blue fluorescent protein. FEMS Microbiology Letters, 329, 61-68. DOI: 10.1111/j.1574-6968.2012.02507.x

Yoshizawa, S., Wada, M., Yokota, A., \& Kogure, K. (2010). Vibrio sagamiensis sp. nov., luminous marine bacteria isolated from sea water. The Journal of General and Applied Microbiology, 56(6), 499-507.

Zhang, X.J., Bi, K.R., Yan, B.L., Qin, L., \& Liang, L.G. (2011). Identification and PCR Detection of Pathogenic Vibrio harveyi Isolated from Gobby Synechogobius hasta. Fishery Science, 30(12), 758-763.

See Digital Appendix at: / Ver Apéndice digital en:

revistas.ucr.ac.cr 